\title{
Repetitive Transcranial Magnetic Stimulation in the Rehabilitation of Vascular Dementia. Report of 2 Cases
}

\author{
Jose Felix Perez Veitia* \\ Neurology Service Cuban Medical Center Mexico. Playa del Carmen, Quintana Roo, Mexico \\ *Corresponding author: José Félix Pérez Veitia, Neurology Service. Clinical Neurology Ave Luis D Colosio 340. Fracc \\ Bosque Real. Playa del Carmen.Quintana Roo. Mexico. CP 77724.
}

\begin{tabular}{l}
\hline ARTICLE INFO \\
\hline Received: 慧 June 12, 2019 \\
Published: 幽 June 21, 2019 \\
Citation: Jose Felix Perez Veitia. Repet- \\
itive Transcranial Magnetic Stimulation \\
in the Rehabilitation of Vascular Demen- \\
tia. Report of 2 Cases. Biomed J Sci \& Tech \\
Res 19(1)-2019. BJSTR. MS.ID.003241.
\end{tabular}

Keywords: Repetitive Transcranial Magnetic Stimulation; Vascular Dementia; Neuromodulation; Neurorehabilitation
SUMMARY

Introduction: Vascular dementia is a clinical syndrome that includes a wide range of cognitive spectrum caused by cerebrovascular disease. Different manifestations and risk factors have been reported in several publications. With the use of transcranial magnetic stimulation has been addressed and deepened in the study of this entity. With the development of the new repetitive magnetic stimulation equipment, the possibility of neuromodularity has been opened and the cortico-subcortical damage present has been rehabilitated, which constitutes a new window into neurological therapeutics.

Clinical Cases: We present two patients with vascular dementia, according to clinical and tomographic criteria. Which present cognitive alterations accompanied by behavioral disorders. Standardized tests are performed and subjected to treatment with repetitive transcranial magnetic stimulation, using dementia and cerebrovascular disease protocols of the Actipulse PRO team. The patients were submitted to 40 sessions. Two months later they undergo the same diagnostic tests and confirming cognitive improvement and executive functions. it was also demonstrated the improvement in the coexistence and social performance.

Conclusion: Repetitive transcranial magnetic stimulation is a new method that is currently revolutionizing the treatment of some neurological and psychiatric diseases. Although its way of acting and duration of treatment is not yet standardized, it gives us a new form of neuromodular brain activity, and it is necessary to deepen new population studies to protocolize treatments.

\section{Introduction}

Vascular Dementia (DV) is a clinical entity that encompasses a broad spectrum of cognitive disorders caused by Cerebrovascular Disease (CVD). The development of Transcranial Magnetic Stimulation (TMS) as a non-invasive study has enabled the study of the cerebral cortex to form a neurophysiological profile of DV. These studies have confirmed the existence of a pattern of cortical hyperexcitability probably related to the alteration of the integrity of white matter lesions due to cerebrovascular disease [1].

The prevalence of dementing syndrome in Latin America and the Caribbean is high, between 6.0 and 6.5 per 100 adults aged 60 and over. The annualized dementia incidence rate standardized for age is also high, with an estimated 410938 new cases of dementia per year, which is associated with lower survival in relation to highincome countries. Of 3, 4 million people with dementia in Latin America and the Caribbean today, the figure will increase to 4.1 million for 2020 and 9.1 million in 2040, that is, it will be similar to that of North America.

Western Europe and North America, have the highest prevalences of dementia in the population of 60 years or older (7.2 and $6.9 \%$ respectively), followed by the Insular Caribbean (6.5\%) and Latin America (6.0\%). The prevalence of dementia per 100 adults over 60 years of age, the estimated number of people with dementia and new cases per year, as well as the proportion of increase in the next four decades and the total costs caused by dementias $[2,3]$. 
DV is reported as the second cause of cognitive impairment in the elderly, being surpassed only by Alzheimer's Disease (AD), some studies report the superposition of both tables [4,5]. It has been shown that CVD risk factors, such as hypertension, atrial fibrillation, diabetes, hypercholesterolemia, atherosclerosis and the apolipoprotein e4 allele, increase the risk of $\mathrm{AD}[6,7]$. The deficit in cholinergic neuronal markers and the decrease in serotonin metabolism observed in $\mathrm{AD}$ have also been associated with DV $[8,9]$. However, DV and EA can be distinguished clinically by the mode of onset and progression of cortical deficits. While memory and language deficits prevail in $\mathrm{AD}$, executive function is more affected in DV, this has been associated with the interruption of frontal networks [10]. Changes in mood and personality occur earlier and are more severe in DV than in AD [11].

Transcranial Magnetic Stimulation (TMS) created as a neurophysiological noninvasive method for assessing the primary motor cortex and corticospinal tract, has changed its application and described the pulses in trains of TMS, are the principle of Repetitive TMS ( RTMS), an approach that can transiently influence the function of the stimulated areas of the brain, according to the frequency of stimulation. RTMS in recent years has been widely recommended in the treatment of neurological and psychiatric diseases [12,13].

In Mexico, a group of researchers have developed an EMT (Actipulse) team, which contributes to increasing the possibilities of neurorehabilitation in our patients. Given the novelty of this treatment, we present the result of the use of rTMS in the treatment of DV.

\section{Case Report}

\section{Clinical Case 1}

It is an 80-year-old female patient with a history of high blood pressure, for which she takes treatment. Go to consultation for a picture of dizziness that exacerbates the movements. He refers to the family that for months has come with behavioral alterations, incorporates stereotyped movements, repetition of sentences. Difficulty remembering names and recent events.

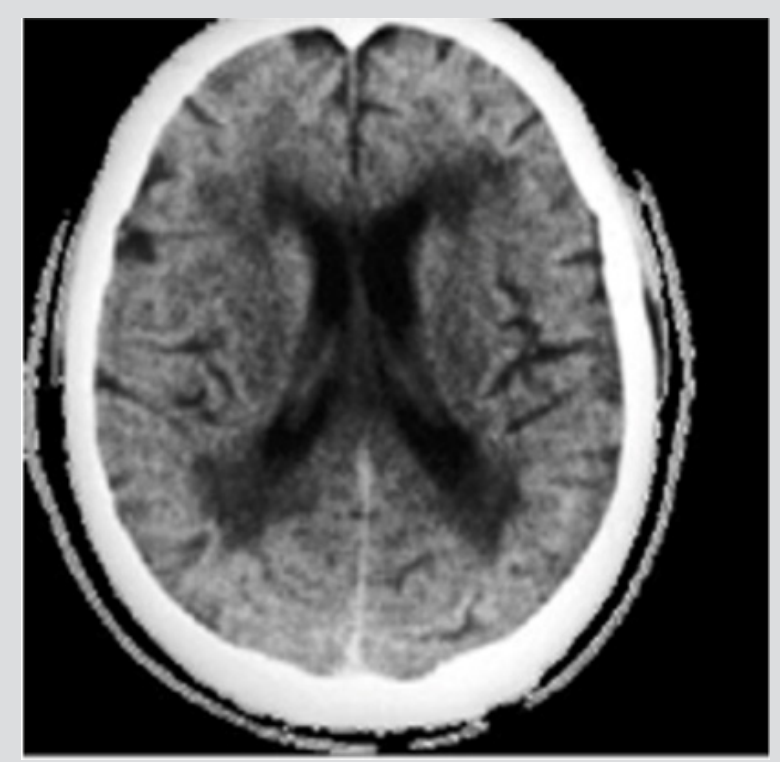

Figure 1: CT scan of the patient's simple skull 1. Subcortical cortical atrophy and presence of leukoatrophy with dilation of the ventricular system in relation to subcortical ischemia.

The neurological examination presents discrete space-time disorientation, with Mini mental test 14 points. Presence of discinence in both hands. Light echolalia. CT scan of the skull with the presence of subcortical cortical atrophy, with increased volume of lateral ventricles due to leukoatrophy (Figure 1). Based on the clinical and tomographic findings, the patient was diagnosed with moderate mild Dementia secondary to cerebrovascular disease subcortical ischemia. After receiving information and consent from the patient, 40 rTMS sessions were applied, using the Dementia and Vascular Brain Disease protocol using the Actipulse equipment, developed in Mexico.

Evaluated the patient at 2 months, relatives report improvement of interpersonal relationships. It does not present language difficulties. Improvement of disorientation, with results of Mental MIni test of 21 points.

\section{Clinical Case 2}

Patient of 75 years, female, history of hypertension. No toxic habits. She refers family members who see her with memory problems for months. The places forget it. has been in recent weeks aggressive with family, isolated, does not speak, remains for hours in bed or sitting with indifference to the environment. Upon physical examination, attention is called to the presence of tremor of the hands and discrete hemiparesis of right hemisphere to brachial predominance. Mini mental test 12 points. CT scan of the skull with the presence of frontal parietal ischemic area. 
Vascular Dementia is diagnosed in this patient and medical treatment is started. The rTMS protocol, the Dementia protocol and the cerebrovascular disease of the Actipulse team were started (Figure 2). Evaluation of the patient at 2 months, we see a patient who integrates into a family environment, performs household chores. Cooperating and socializing with their environment. Mini mental test 22 points.

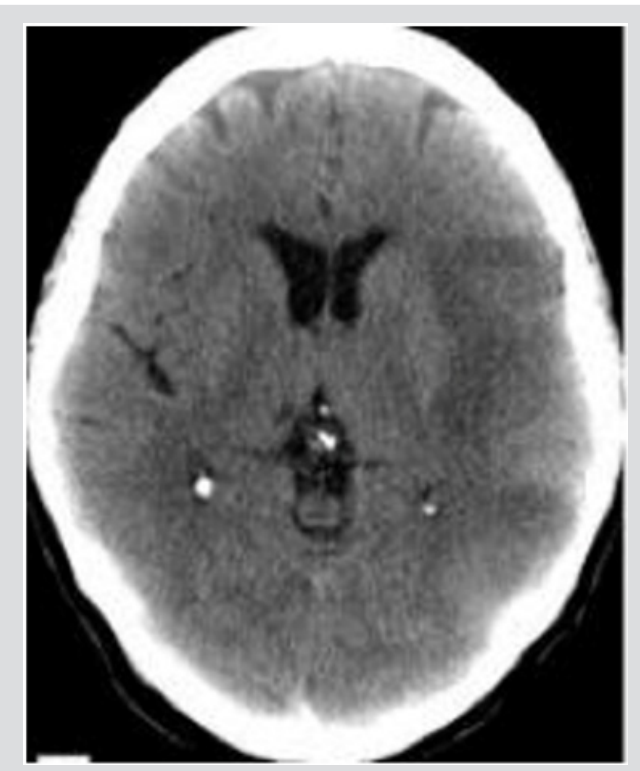

Figure 2: CT scan of the simple skull of patient 2. A hypodense image is observed in relation to left-sided cerebral artery ischemic CVD, which affects the left parietal fronto region.

\section{Discussion}

RTMS has been described as a technique that interferes with cerebral Neuroplasticity (NP) with improvement of vascular sequelae [14-17]. Several publications have highlighted the effects of rTMS on NP, which may have a dual role in facilitating or inhibiting neuronal synaptic connections. Most of the clinic generated after the stroke is not due to the injury itself, but to the hyperactivity recorded in the intact hemisphere, which indirectly inhibits the injured. It is described that the low frequency rTMS ( $\leq 1 \mathrm{~Hz}$ ) applied to the healthy hemisphere normalizes the diffuse cortical activation of the primary and secondary motor areas of both cerebral hemispheres, reactivating the injured cortical area that had been inhibited, favoring its excitability and motor recovery. However, high frequency rTMS ( $\geq 5 \mathrm{~Hz}$ ) increases cortical excitability and can be applied to produce a neuronal stimulation of the cerebral cortex of the injured hemisphere. That is, rTMS accelerates NP mechanisms, reorganizing brain connections, which leads to greater efficiency of the interneuronal networks of the affected brain area [18-21].

EMT has been used in investigations of motor pathways, changes in corticocortical circuits after CVD and with special interest as a potential therapy that promotes reorganization and improves the response to conventional treatments [22]. The rTMS given its principles has potential in the reactivation of the cerebral stroke. Human studies show the potential of the cortical regions adjacent to the injured area that contribute to recovery by functional remodeling of the motor cortex representations. It has been reported that rTMS modulates neural excitability through its action on non-injured intracortical connections. Motor behavior after CVD is a primary objective of EMT interventions. There is a balance between the function of the two hemispheres, controlled by interhemispheric inhibition. The affected hemisphere may be altered by the CVD itself and by the unbalanced inhibition that the healthy hemisphere generates. In this model, the increased activity of the affected hemisphere promotes the recovery of the diseased hemibody and induces the decrease of inhibition from the healthy hemisphere. For example, it has been found that motor performance after ECV improves after inhibiting the healthy hemisphere with rTMS at a low frequency [23] or stimulating the affected hemisphere with rTMS at high frequency [24].

In attempting to exploit rTMS protocols as a therapeutic option for DV, a randomized controlled pilot study showed that a high frequency rTMS session applied to left DLPFC improved executive performance, whereas no effects were seen in any other cognitive function [25]. The excitatory and inhibitory electromagnetic pulses applied in the cerebral hemisphere ipsilateral or contralateral to the lesion, respectively, as well as at the transcallosus level to modulate cerebral interhemispheric communication, offer us the possibility of optimizing functional brain activity, as well as achieving a recovery of the area damaged The existence of impaired synaptic plasticity in the pathophysiology of vascular dementia suggests a role for rTMS as an adequate neurorehabilitation tool, this has been suggested in different publications $[25,26]$.

\section{Conclusion}

In both cases of our patients we saw evident improvement after the use of rTMS. For the time being, the most adequate duration of 
treatment, the exact moment of intervention and the most suitable protocol are unknown. Surely in the realization of prospective studies, with larger samples of patients and longer follow-up times, it will allow us to obtain a higher level of scientific evidence, which allows us to clarify these doubts and thereby implement rTMS in the process of neurorehabilitation of the patient with acquired brain damage secondary to a stroke, without abandoning other physical and neurocognitive rehabilitation techniques.

\section{References}

1. Pennisi G, Ferri R, Cantone M, Lanza G, Pennisi M, et al. (2011) A review of transcranial magnetic stimulation in vascular dementia. Dementia and Geriatric Cognitive Disorders 31: 71-80.

2. Llibre Rodriguez JJ, Gutierrez R (2014) Dementias and Alzheimer's disease in Latin America and the Caribbean. Cuban Revolution Public Health $40(3)$.

3. Prince M, Guerchet M, Prina M (2013) Policy Brief for Heads of Government: The Global Impact of Dementia 2013-2050. London, p. 1-7.

4. Snowdon DD A, Greiner LH, Mortimer JA, Riley KP, Greiner PA, et al. (1997) Brain infarction and the clinical expression of Alzheimer's disease: The Nun Study. JAMA 277: 813-817.

5. Kalaria R (2002) Similarities between Alzheimer's disease and vascular dementia. J Neurol Sci 203-204: 29-34.

6. Skoog I, Lernfelt B, Landahl S, Palmertz B, Andreasson LA, et al. (1996) 15-year longitudinal study of blood pressure and dementia. Lancet 347: 1141-1145.

7. Premkumar DR, Cohen DL, Hedera P, Friedland RP, Kalaria RN (1996) Apolipoprotein E-4 alleles in cerebral amyloid angiopathy and cerebrovascular pathology associated with Alzheimer's disease. Am J Pathol 148: 2083-2095.

8. Murley AG, Rowe JB (2018) Neurotransmitter deficits from frontotemporal lobar degeneration. Brain 14(5): 1263-1285.

9. Richter N, Michel A, Onur OA, Kracht L, Dietlein M, et al. (2017) White matter lesions and the cholinergic deficit in aging and mild cognitive impairment. Neurobiology of Aging. 53: 27-35.

10. Román GC, Royall DR (1999) Executive control function: A rational basis for the diagnosis of vascular dementia. Alzheimer Dis Assoc Disord 13 S69-S80.

11. Olazarán-Rodríguez J, Agüera-Ortiz LF, Muñiz-Schwochert R (2012) Psychological and behavioral symptoms of dementia: prevention, diagnosis and treatment. Rev Neurol 55: 598-608.

12. Chibbaro G, Daniele M, Alagona G, Di Pasquale C, Cannavò M, et al. (2005) Repetitive transcranial magnetic stimulation in schizophrenic patients reporting auditory hallucinations. Neurosci Lett 383: 54-57.

13. Schönfeldt-Lecuona C, Lefaucheur JP, Cardenas-Morales L, Wolf RC, Kammer T, et al. (2010) The value of neuronavigated rTMS for the treatment of depression. Neurophysiol Clin 40: 37-43.

\section{ISSN: 2574-1241}

\section{DOI: 10.26717/BJSTR.2019.19.003241}

Jose Felix Perez Veitia. Biomed J Sci \& Tech Res

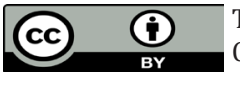

This work is licensed under Creative Commons Attribution 4.0 License

Submission Link: https://biomedres.us/submit-manuscript.php
14. Pennisi G, Alagona G, Ferri R, Greco S, Sanotonito D, et al. (2002) Motor cortex excitability in Alzheimer's disease: one-year follow-up study. Neurosci Lett 329: 293-296.

15. Di Lazzaro V, Oliviero A, Tonali PA, Marra C, Daniele A, et al. (2002) Noninvasive in vivo assessment of cholinergic cortical circuits in AD using transcranial magnetic stimulation. Neurology 59: 392-397.

16. Di Lazzaro V, Pilato F, Oliviero A, Dileone M, Saturn E, et al. (2006) Origin of facilitation of motor evoked 77 potentials after paired magnetic stimulation: direct recording of epidural activity in conscious humans. J Neurophysiol 96: 1765-1771.

17. Wang F, Geng X, Tao HY, Cheng Y (2010) The restoration after repetitive transcranial magnetic stimulation treatment on cognitive ability of vascular dementia rats and it's on synaptic plasticity in hippocampal CA1 area. J Mol Neurosci 41: 145-155.

18. Escribano MB, Tunisia I (2015) Transcranial magnetic stimulation as a new therapeutic strategy in stroke. In: Castillo Sánchez J, Jiménez Martín I (Eds.). Functional reeducation after a stroke. Barcelona: Elsevier Spain, SLU pp. 121-133.

19. Auriat AM, Neva JL, Peters S, Ferris JK, Boyd LA (2015) A review of transcranial magnetic stimulation and multimodal neuroimaging to characterize post-stroke neuroplasticity. Front Neurol 6: 226.

20. Pascual-Leone A, Tormos- Muñoz JM (2008) Transcranial magnetic stimulation: Fundamentals and potential of the modulation of specific neuronal networks. Rev Neurol 46: S3-S10.

21. Turkeltaub PE (2015) Brain stimulation and the role of the right hemisphere in aphasia recovery. Curr Neurol Neurosc Rep 15: 72.

22. Claflin ES, Krishnan C, Khot SP (2015) Emerging treatments for motor rehabilitation after stroke. Neurohospitalist 5: 77-88.

23. Kobayashi M, Pascual-Leone A (2003) Transcranial magnetic stimulation in neurology. Lancet Neurol 2: 145-156

24. Emara TH, Moustafa RR, Elnahas NM, Elganzoury AM, Abdo TA, et al. (2010) Repetitive transcranial magnetic stimulation at $1 \mathrm{~Hz}$ and 5 $\mathrm{Hz}$ used to improve the motor function and disability after ischaemic stroke. Eur J Neurol 17: 1203-1209.

25. Bayón M (2011) Transcranial magnetic stimulation in the rehabilitation of stroke. Rehabilitation 45: 261-267.

26. Rektorova I, Megova S, Bares M, Rektor I (2005) Cognitive functioning after repetitive transcranial magnetic stimulation in patients with cerebrovascular disease without dementia: a pilot study of seven patients. J Neurol Sci 15: 229-230.

27. Maria Cotelli, Rosa Manenti, Orazio Zanetti, Carlo Miniuss (2012) Nonpharmacological intervention for memory decline. Frontiers in Human Neuroscience 6 (46): 1-17.

28. Gervits F, Ash S, Coslett HB, Rascovsky K, Grossman M, et al. Transcranial direct current stimulation for the treatment of primary progressive aphasia: An open-label pilot study. Brain and Language 16: 35-41.

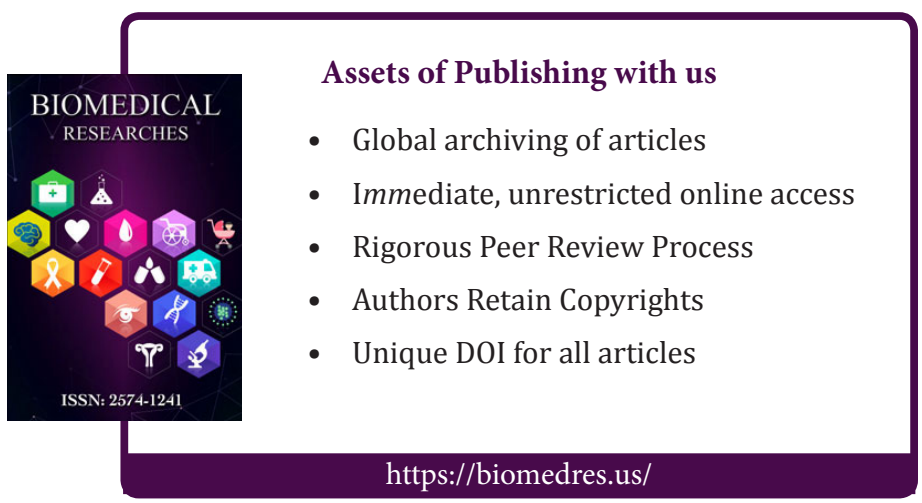

